\title{
Utilização de abacaxi (Ananas comosus L.) cv. Pérola e Smooth cayenne para a produção de vinhos - estudo da composição química e aceitabilidade
}

\author{
Utilization of pineapple (Ananas comosus L.) cv. Pérola and Smooth \\ cayenne for wine making - study of chemical composition and acceptance
}

\author{
Kátia Gomes Lima ARAÚJO ${ }^{1}$, Armando Ubirajara Oliveira SABAA-SRUR ${ }^{2,3}$, \\ Fabiana Silva RODRIGUES ${ }^{3 *}$, Luciana Ribeiro Trajano MANHÃES ${ }^{3,4}$, Marta Weber do CANTO 4
}

\begin{abstract}
Resumo
Foram estudadas as características químicas de vinhos produzidos com as cultivares de abacaxi Pérola e Smooth cayenne, com vistas a predizer a qualidade das bebidas, tomando-se como base a sua composição e aceitabilidade. Os mostos de abacaxi foram analisados quanto ao pH, acidez total, ácidos orgânicos fixos, açúcar total, pectina total, compostos fenólicos, $\mathrm{K}, \mathrm{Ca}, \mathrm{Fe}, \mathrm{Cu}, \mathrm{N}$ e $\mathrm{P}$, enquanto nos vinhos analisou-se também a acidez volátil, teor alcoólico, alcoóis superiores, acetaldeído, acetato de etila, glicerol, metanol e cor. Os resultados indicaram que existem semelhanças em relação à composição química de mostos e vinhos de abacaxis e uvas. A análise dos compostos voláteis formados durante a fermentação alcoólica indicou que a composição do mosto de abacaxi interferiu nas quantidades de alguns voláteis produzidos pela levedura Saccharomyces cerevisiae. O teste de aceitabilidade com escala hedônica de nove pontos indicou que os vinhos elaborados com as duas cultivares de abacaxi foram igualmente aceitos.
\end{abstract}

Palavras-chave: abacaxi; fermentação alcoólica; vinho; composição; aceitabilidade.

\begin{abstract}
The objective of the present work was to study the chemical characteristics of wine made with two different varieties of pineapple: Pérola and Smooth cayenne, in order to predict its quality based on composition and acceptance. The pineapple musts were chemically characterized through the analysis of $\mathrm{pH}$, total acidity, fixed organic acids, total sugar, total pectin, phenolic compounds, and $\mathrm{K}$, Ca, $\mathrm{N}$ e P. The wines were characterized by volatile acidity, alcoholic grade, higher alcohols, acetaldehyde, ethyl acetate, glycerol, methanol, and color. The results showed that there are similarities between the pineapple and grape musts and wines. The analysis of volatile compunds performed during the alcoholic fermentation showed that the pineapple must composition interfered with the quantities of volatiles formed by Saccharomyces cerevisiae. The 9-point hedonic scale acceptance indicated that the two varieties of wine were accepted by the panel.

Keywords: pineapple; alcoholic fermentation; wine; chemical composition; acceptance.
\end{abstract}

\section{Introdução}

O abacaxi é um fruto muito apreciado em várias regiões do mundo, constituindo-se num dos principais produtos da fruticultura nacional. Apesar da abundância do cultivo dessa fruta no Brasil, o aproveitamento industrial ainda é pequeno frente ao consumo da fruta in natura, sendo necessária a busca de alternativas para o seu uso, visando o aproveitamento do excesso de safras, principalmente pela indústria, para a fabricação de produtos não tradicionais, como, por exemplo, de vinhos, em função da concentração de açúcares fermentescíveis, acidez e características sensoriais.

Tradicionalmente, os vinhos são preparados pela fermentação de mostos de uvas, que produzem etanol e outros produtos metabólicos. No entanto, a literatura cita a produção de vinho a partir de outras frutas como amora (AMERINE; BERG; CRUESS, 1972), abacaxi (ALIAN; MUSSENGE, 1976; MALDONADO; ROLZ; CABRERA, 1972), manga (ONKARAYYA; SINGH, 1984), kiwi (WITH; LODGE, 1982), melão (BENK, 1987), maracujá (BENK, 1987), etc. Segundo Cantareli (1982), a produção de vinho de abacaxi é quase que exclusivamente caseira, podendo atingir escala maior desde que encontre mercado consumidor. Pode ser obtido em boas condições se forem usadas as mesmas técnicas adotadas para a fermentação de mostos de uvas.

Quanto à composição química, o vinho é uma bebida extremamente complexa, contendo substâncias das mais variadas classes. Os compostos voláteis secundários da fermentação

\footnotetext{
Recebido para publicação em 20/6/2007

Aceito para publicação em 3/1/2009 (002598)

${ }^{1}$ Departamento de Bromatologia, Faculdade de Farmácia, Universidade Federal Fluminense - UFF, Rua Mário Viana, 523, Santa Rosa, CEP 24241-000, Niterói - RJ, Brasil

2 Grupo de Pesquisa em Processamento de Frutas e Hortaliças - GPPFH, Departamento de Nutrição Básica e Experimental - DNBE,

Instituto de Nutrição Josué de Castro - INJC, Universidade Federal do Rio de Janeiro - UFRJ, Av. Carlos Chagas, 373, Ilha do Fundão, CEP 21941-902, Rio de Janeiro - RJ, Brasil

${ }^{3}$ Grupo de Pesquisa em Processamento de Frutas e Hortaliças - UFRJ, Instituto de Nutrição Josué de Castro - INJC, Departamento de Nutrição Básica e Experimental -

DNBE, Universidade Federal do Rio de Janeiro - UFRJ, Av. Carlos Chagas, 373, Ilha do Fundão - RJ, CEP 21941-902, Brasil, E-mail: fabiananutri@hotmail.com

${ }^{4}$ Departamento de Tecnologia e Ciência dos Alimentos, Universidade Federal de Santa Maria - UFSM, Campus COMOBI, CEP 97105-900, Santa Maria - RS, Brasil

${ }^{*}$ A quem a correspondência deve ser enviada
} 
alcoólica (alcoóis superiores, acetato de etila e acetaldeído) interferem diretamente na qualidade do aroma (NYKANEN, 1986) e o glicerol, também subproduto da fermentação, contribui para a viscosidade, doçura e corpo dos vinhos (LAGE et al., 1989; NOBLE; BURSICK, 1984). A presença de metanol está relacionada aos teores de pectina dos mostos (GNEKOW; OUGH, 1976). Os compostos fenólicos, particularmente os flavonóides, conferem atributos de amargor e adstringência, sendo também responsáveis pela precipitação de complexos de proteínas e pelo escurecimento de vinhos brancos (ARNOLD; NOBLE; SINGLETON, 1980). Minerais como o potássio e o cálcio podem originar a formação de depósitos de sais de ácido tartárico em vinhos engarrafados. $\mathrm{O}$ ferro interfere na estabilidade física pela precipitação de fosfato ferroso (casse branca) e complexos com taninos (casse azul) (ZOECKLEIN, 1989), e o cobre, por sua vez, pode originar a precipitação de sulfeto de cobre (casse cúprica) (COPPOLA, 1982).

O objetivo do presente trabalho foi a produção de vinhos de abacaxi e a caracterização da sua composição química, como índice de sua qualidade, comparando-os com vinhos de uvas, bem como a determinação da sua aceitabilidade.

\section{Material e métodos}

Foram utilizados abacaxis (Ananas comosus L.) das cultivares Pérola e Smooth cayenne. A fermentação alcoólica foi conduzida com cepas de Saccharomyces cerevisiae 79 - B, oriunda do Centro Nacional de Pesquisa de Uvas e Vinhos - EMBRAPA do município de Bento Gonçalves - RS

\subsection{Obtenção dos mostos e vinhos}

Os frutos depois de lavados e descascados foram desintegrados e os sucos obtidos foram centrifugados a $5^{\circ} \mathrm{C}$, a $5.500 \mathrm{rpm}$ por 20 minutos. Aos sobrenadantes, foram adicionados $100 \mathrm{mg} . \mathrm{L}^{-1}$ de anidrido sulfuroso e tiveram seus conteúdos de açúcar corrigidos com sacarose até a concentração de sólidos solúveis de $20 \%$; para cada $\mathrm{L}$ de suco integral de abacaxi, foram adicionados aproximadamente $70 \mathrm{~g}$ de açúcar. Cerca de 5,0 L de mosto recebeu a adição de $3 \%$ (v/v) de pé-de-cuba em plena fermentação que foi preparada pela inoculação de cepas de Saccharomyces cerevisiae 79 - B em suco de abacaxi previamente esterilizado a $121,1{ }^{\circ} \mathrm{C}$ por 30 minutos e incubado a $19^{\circ} \mathrm{C}$ sob agitação de $250 \mathrm{rpm}$. A temperatura da fermentação alcoólica foi mantida entre $18-20^{\circ} \mathrm{C}$ por 8 dias, sendo o final do processo definido em função da estabilização da concentração residual de açúcares redutores no mosto fermentado. A fermentação alcoólica foi realizada em 4 repetições.

Quinze dias após o término do processo fermentativo, os vinhos mantidos sob refrigeração foram trasfegados e receberam a adição de $300 \mathrm{mg} . \mathrm{L}^{-1}$ de bentonite, preparada segundo Daudt e Durante (1986). A estabilização dos vinhos ocorreu por 7 meses sob refrigeração $\left(0-3^{\circ} \mathrm{C}\right)$, realizando-se trasfegas periódicas com a adição de anidrido sulfuroso até a concentração de $50 \mathrm{mg} \cdot \mathrm{L}^{-1}$. Os vinhos foram então acondicionados em garrafas de vidro âmbar, fechadas sob pressão com rolhas de cortiça, armazenados à temperatura ambiente e analisados. Cada embalagem continha aproximadamente $750 \mathrm{~mL}$ de vinho.

\subsection{Métodos analíticos}

$\mathrm{O}$ pH foi medido em potenciômetro digital. Análises de acidez total e volátil foram feitas segundo Amerine e Ough (1974). Os ácidos orgânicos fixos (cítrico, málico, oxálico e succínico) foram dosados por cromatografia líquida de alta eficiência segundo AOAC (1986). Os açúcares redutores e totais foram determinados pelo método de Lane-Eynon (1974). A cromatografia em fase gasosa foi utilizada para a dosagem do etanol (ROBBS; SANTANA; GULARTE, 1983), glicerol (LAGE, 1989) e compostos voláteis (acetaldeído, acetato de etila, n-propanol, isobutanol, alcoóis amílicos e metanol) (CANTARELLI, 1982). Para a dosagem da pectina foi utilizado o método de McCready e McComb (1952) para a extração e o método de Bitter e Muir (1962) para a quantificação. Os compostos fenólicos totais foram dosados pelo método de Folin-Ciocalteau (1974) e os não flavonóides, segundo a técnica de Kramling e Singleton (1969). A cor dos vinhos de abacaxi foi avaliada pela absorbância a $420 \mathrm{~nm}$ (AMERINE; OUGH, 1974). O potássio, cálcio, ferro e cobre, foram analisados por espectrometria de absorção atômica em chama após mineralização da amostra por via úmida (GONZALEZ; PARA; AQUILAR, 1988). O fósforo foi analisado segundo a técnica descrita no IUPAC (1979) e o nitrogênio total pelo método de microkjeldahl (AOAC, 1986).

\subsection{Aceitabilidade dos vinhos de abacaxi}

A aceitabilidade dos produtos foi realizada no Departamento de Tecnologia de Alimentos da Universidade Federal Rural do Rio de Janeiro e avaliada por um grupo de 73 provadores não treinados, que receberam cerca de $50 \mathrm{~mL}$ de amostras servidas em taças de vidro apropriadas para esse tipo de bebida, sendo utilizada escala hedônica de nove pontos (SIMPSON, 1979). A análise estatística da preferência entre as cultivares foi efetuada pela análise da variância, segundo Chaves (1980).

\section{Resultados e discussão}

A composição química dos mostos e vinhos de abacaxi é apresentada nas Tabelas 1 e 2, respectivamente.

Os valores de $\mathrm{pH}$ e acidez total dos mostos e dos vinhos de abacaxi estavam de acordo com aqueles relatados na literatura para mostos e vinhos de uvas (GUINARD; PANGBORN; LEWIS, 1986; MORALEDA, 1988). Depois do etanol, os ácidos orgânicos são as substâncias que mais contribuem para o sabor dos vinhos. A acidez destas bebidas procede tanto do mosto quanto da fermentação alcoólica, de modo que o $\mathrm{pH}$ final situa-se normalmente entre 2,0 e 4,0. Valores de $\mathrm{pH}$ acima de 4,0 tornam os vinhos sujeitos a alterações microbiológicas e de cor. A acidez volátil dos vinhos de abacaxi indicou que a concentração de ácido acético não estava presente em quantidade capaz de depreciar sua qualidade sensorial. Em vinhos de uvas, segundo Drysdale e Fleet, concentrações de até 2,0 g.L $\mathrm{L}^{-1}$ são suficientes para aviltar sensorialmente esses vinhos (DRYSDALE; FLEET, 1985).

Foi observado que diferentes concentrações de alcoóis superiores totais foram formadas durante a fermentação alcoólica dos mostos, em função da cultivar do abacaxi, sendo que os maiores teores dos alcoóis n-propílico, isobutílico e amílicos foram detectados no vinho de abacaxi da cv Pérola. Atualmente, 
dois mecanismos são propostos para explicar a formação dos alcoóis superiores pelas leveduras. Por via catabólica, a partir dos aminoácidos do meio fermentativo, e por via anabólica, a partir dos carboidratos, durante a síntese de aminoácidos pela célula (COPPOLA, 1982). Lee e Cooley (1987) relataram que a formação dos alcoóis superiores pode ser dependente do nitrogênio presente no mosto, por estimular o consumo dos aminoácidos e a sua síntese a partir dos carboidratos. Os resultados deste trabalho mostraram maior formação de alcoóis superiores no vinho de abacaxi cujo mosto possuía maior teor de nitrogênio total.

Os odores dos alcoóis superiores são descritos como "alcoólicos, doces e pesados”, reforçando o aroma vegetal e herbáceo do n-hexanol das uvas (BERTRAND, 1981). A presença dos alcoóis superiores em vinhos, nas concentrações de 140 a 420 mg. $\mathrm{L}^{-1}$, exerce um papel favorável à sua qualidade sensorial, no entanto, teores elevados tornam o aroma "asfixiante", depreciando a sua qualidade (BERTRAND, 1981; ZOECKLEIN et al., 1989). Os teores médios de alcoóis superiores encontrados para os vinhos de abacaxi (Tabela 2) são concordantes com os apresentados na literatura para vinhos de uvas, cuja variação é de 6-63 mg. $\mathrm{L}^{-1}$ para o n-propílico, 12-174 mg. $\mathrm{L}^{-1}$ para o isobutílico e 55-384 mg. $\mathrm{L}^{-1}$ para os alcoóis amílicos (LEE; COOLEY, 1981; RIZZON, 1987; SHINOHARA; WATANABE, 1976; SIMPSON, 1979), com excesso de álcool n-propílico do vinho de abacaxi da cultivar Pérola, que foi superior à faixa apresentada. As concentrações dos alcoóis superiores totais nos vinhos de abacaxi das cultivares Pérola e Smooth cayenne (respectivamente, 404,0 \pm 9,6 e $347,6 \pm$ 9,4 mg. $\mathrm{L}^{-1}$ ) incluíram-se na faixa em que estes alcoóis favorecem o aroma de vinhos de uvas. Provavelmente, o efeito no aroma do vinho de abacaxi da cv. Pérola seja mais acentuado que na cv Smooth cayenne, por aquele apresentar 16,2\% a mais de alcoóis superiores totais.

O conteúdo de acetato de etila no vinho de abacaxi da cv. Pérola foi maior que no da cv. Smooth cayenne (Tabela 2). Ough e Lee (1981) relacionaram a formação de ésteres durante a fermentação alcoólica aos conteúdos de nitrogênio total de mostos de uvas, supondo que a resposta metabólica da levedura difere em função da composição do meio. Visto que esse experimento foi realizado em condições constantes, diferenciados apenas pelos cultivares dos abacaxis utilizados na obtenção dos mostos, a formação do acetato de etila foi dependente da composição do mosto, fator este que pode influenciar a qualidade e intensidade do aroma dos vinhos de abacaxi. Os teores médios deste éster nos vinhos foram comparáveis às faixas apresentadas por Rizzon (1984) para 75 vinhos brancos $\left(26,9-173,2 \mathrm{mg} . \mathrm{L}^{-1}\right)$ e tintos (49,7-208,1 mg.L. $\left.\mathrm{L}^{-1}\right)$. As condições experimentais utilizadas não induziram a formação do acetato de etila nos vinhos de abacaxi em quantidades superiores a $200 \mathrm{mg} . \mathrm{L}^{-1}$, citadas como capazes de conferir características de deterioração ao aroma de vinhos de uvas (AMERINE; BERG; CRUESS, 1972; RIZZON, 1987).

Os teores médios do acetaldeído nos vinhos de abacaxi (Tabela 2) não atingiram valores superiores a $100 \mathrm{mg} . \mathrm{L}^{-1}$, citados como limite inferior para a sua percepção pelo olfato em vinhos (ALIAN; MUSSENGE, 1976), e enquadraram-se na faixa apresentada por Nykanen (1986) para vinhos brancos (13-108 mg.L $\left.\mathrm{L}^{-1}\right)$. O teor médio do metanol no vinho de abacaxi da cultivar Smooth cayenne foi maior que no da cultivar
Pérola (Tabela 2), o que foi relacionado ao maior conteúdo de pectina presente no mosto da cultivar Smooth cayenne (Tabela 1). Estes resultados são concordantes com os de San Miguel (1982) e Nykanen (1986), que relataram que o metanol

Tabela 1. Composição dos mostos de abacaxi das cv. Pérola e Smooth Cayenne.

\begin{tabular}{|c|c|c|}
\hline & Pérola & Smooth cayenne \\
\hline $\mathrm{pH}$ & $3,77 \pm 0,01$ & $3,55 \pm 0,01$ \\
\hline Acidez total (g ac. cítrico/L) & $3,9 \pm 0,1$ & $5,7 \pm 0,1$ \\
\hline \multicolumn{3}{|l|}{ Ácidos orgânicos (mg. $\left.\mathrm{L}^{-1}\right)$} \\
\hline Cítrico & $4381,8 \pm 10,0$ & $6164,8 \pm 8,1$ \\
\hline Málico & $2097,5 \pm 10,0$ & $1922,3 \pm 21,1$ \\
\hline Oxálico & $963,4 \pm 8,5$ & $882,9 \pm 7,3$ \\
\hline Succínico & $146,0 \pm 8,1$ & $159,3 \pm 6,9$ \\
\hline Açúcar total ${ }^{1}$ (g.100 mL $\left.\mathrm{mL}^{-1}\right)$ & $19,3 \pm 0,9$ & $19,0 \pm 0,5$ \\
\hline Pectina total (g.L $\left.\mathrm{L}^{-1}\right)$ & $1,1 \pm 0,05$ & $2,2 \pm 0,05$ \\
\hline \multicolumn{3}{|c|}{ Compostos fenólicos (mg.L $\left.\mathrm{L}^{-1} \mathrm{EAG}^{2}\right)$} \\
\hline Flavonóides & $308,5 \pm 0,1$ & $183,6 \pm 0,5$ \\
\hline Não flavonóides & $491,4 \pm 0,1$ & $273,1 \pm 0,1$ \\
\hline \multicolumn{3}{|l|}{ Minerais (mg. $\left.\mathrm{L}^{-1}\right)$} \\
\hline K & $1301,6 \pm 4,5$ & $936,2 \pm 3,2$ \\
\hline $\mathrm{Ca}$ & $67,4 \pm 1.1$ & $76,2 \pm 3,2$ \\
\hline $\mathrm{Fe}$ & $3,01 \pm 0,01$ & $2,12 \pm 0,01$ \\
\hline $\mathrm{Cu}$ & $1,33 \pm 0,11$ & $1,12 \pm 0,05$ \\
\hline $\mathrm{N}$ & $914,0 \pm 1,0$ & $590,0 \pm 1,0$ \\
\hline $\mathrm{P}$ & $83,6 \pm 2,0$ & $56,4 \pm 1,2$ \\
\hline
\end{tabular}

Tabela 2. Composição dos vinhos de abacaxi das cultivares Pérola e Smooth cayenne.

\begin{tabular}{|c|c|c|}
\hline & Pérola & Smooth cayenne \\
\hline $\mathrm{pH}$ & $3,68 \pm 0,01$ & $3,47 \pm 0,01$ \\
\hline Acidez total (g ác. cítrico/L) & $4,77 \pm 0,03$ & $6,55 \pm 0,09$ \\
\hline Acidez volátil (g ác. acético/L) & $0,42 \pm 0,01$ & $0,39 \pm 0,01$ \\
\hline \multicolumn{3}{|l|}{ Ácidos orgânicos $\left(\mathrm{mg} . \mathrm{L}^{-1}\right)$} \\
\hline Cítrico & $4125,0 \pm 24,1$ & $5870,9 \pm 20,4$ \\
\hline Málico & $1982,5 \pm 41,8$ & $1853,7 \pm 42,2$ \\
\hline Oxálico & $675,9 \pm 19,6$ & $600,7 \pm 28,3$ \\
\hline Succínico & $1322,4 \pm 31,5$ & $944,9 \pm 72,3$ \\
\hline Açúcares redutores (g glicose/L) & $0,31 \pm 0,01$ & $0,30 \pm 0,01$ \\
\hline Etanol $\left({ }^{\circ} \mathrm{GL}\right)$ & $11,2 \pm 0,2$ & $10,9 \pm 0,3$ \\
\hline \multicolumn{3}{|l|}{ Alcoóis superiores (mg. $\mathrm{L}^{-1}$ ) } \\
\hline N-Propanol & $76,4 \pm 2,4$ & $56,2 \pm 2,4$ \\
\hline Isobutanol & $98,5 \pm 2,0$ & $88,2 \pm 0,7$ \\
\hline Alcoóis amílicos & $229,1 \pm 2,4$ & $202,8 \pm 3,9$ \\
\hline Metanol (mg. $\left.L^{-1}\right)$ & $20,1 \pm 1,5$ & $43,2 \pm 1,9$ \\
\hline Acetaldeído (mg.L $\left.\mathrm{L}^{-1}\right)$ & $48,1 \pm 1,3$ & $44,6 \pm 0,4$ \\
\hline Acetato de etila (mg. $\left.\mathrm{L}^{-1}\right)$ & $71,2 \pm 5,7$ & $42,6 \pm 3,6$ \\
\hline \multicolumn{3}{|l|}{ Compostos fenólicos (mg.L $\left.\mathrm{L}^{-1} \mathrm{EAG}\right)$} \\
\hline Flavonóides & $106,8 \pm 1,0$ & $35,8 \pm 0,3$ \\
\hline Não flavonóides & $325,6 \pm 0,9$ & $132,6 \pm 1,2$ \\
\hline Cor (A $420 \mathrm{~nm})$ & $0,314 \pm 0,006$ & $0,197 \pm 0,014$ \\
\hline Glicerol (g.L $\left.\mathrm{L}^{-1}\right)$ & $6,9 \pm 0,4$ & $6,4 \pm 0,3$ \\
\hline \multicolumn{3}{|l|}{ Minerais } \\
\hline K & $1265,4 \pm 1,8$ & $916,3 \pm 1,5$ \\
\hline $\mathrm{Ca}$ & $57,9 \pm 1,3$ & $61,9 \pm 1,2$ \\
\hline $\mathrm{Fe}$ & $3,71 \pm 0,3$ & $3,06 \pm 0,1$ \\
\hline $\mathrm{Cu}$ & $0,34 \pm 0,03$ & $0,45 \pm 0,02$ \\
\hline $\mathrm{N}$ & $460,7 \pm 14,1$ & $384,8 \pm 8,6$ \\
\hline $\mathrm{P}$ & $23,6 \pm 1,4$ & $7,3 \pm 0,4$ \\
\hline
\end{tabular}


origina-se nos vinhos a partir da desmetilação enzimática da pectina. Os teores de metanol nos vinhos de abacaxi foram similares aos encontrados por Gnekow e Ough (1985) para vinhos brancos (18,9-40,5 mg. $\left.\mathrm{L}^{-1}\right)$ e inferiores para vinhos tintos (61,3-155,6 mg. $\left.\mathrm{L}^{-1}\right)$.

A Tabela 1 indica que os mostos das duas cultivares de abacaxi foram mais ricos em fenóis não flavonóides. Somers e Zimelins (1985) e Noble (1990) relataram a predominância de flavonóides em suco de uva branca (cerca de 200 mg.L $\mathrm{L}^{-1}$, em equivalentes de ácido gálico (E.A.G.)), mais próximo do teor encontrado no mosto da cultivar Smooth cayenne. As diminuições observadas nos teores de fenóis durante a vinificação são devidas à sua precipitação sob a forma de complexos proteico-fenólicos, que concorre para a clarificação dos vinhos (NOBLE, 1990; ZOECKLEIN et al., 1989). Os teores de fenóis totais determinados nos vinhos de abacaxi são concordantes com os determinados em vinhos brancos por Kramling e Singleton (1969) e Ohkubo (1984) (151-455 mg.L ${ }^{-1}$ E.A.G.). Provavelmente, o maior conteúdo de compostos fenólicos encontrados no vinho de abacaxi variedade Pérola concorra para torná-lo mais amargo e adstringente que o Smooth cayenne e, também, seja responsável pela maior oxidação química e/ ou enzimática observada através da determinação da cor dos vinhos (Tabela 2). Amostras de vinhos brancos analisados por Ohkubo et al. (1984) apresentaram absorbância média a $420 \mathrm{~nm}$ de 0,14 , mais próxima da encontrada no vinho de abacaxi da cultivar Smooth cayenne que no da cultivar Pérola.

Os teores de glicerol (Tabela 2) encontrados nos vinhos de abacaxi estão de acordo com os citados na literatura para vinhos de uvas (1,9-14,7 g.L L $^{-1}$ ) (AMERINE; OUGH, 1974). Noble e Bursik (1990) verificaram que o sabor do glicerol é percebido em vinhos quando a sua concentração é superior a 5,2 g. $\mathrm{L}^{-1}$.

A determinação dos minerais nos mostos e vinhos de abacaxi mostrou que, exceto para o ferro, todos os outros tiveram seus teores diminuídos em relação ao vinho (Tabelas 1 e 2), como também ocorre para vinhos de uvas (ZOECKLEIN, 1989). Possivelmente, as eliminações observadas nos conteúdos de potássio e cálcio na vinificação dos mostos de abacaxi tenham sido decorrentes da sua precipitação sob a forma de sais de ácidos orgânicos, o que foi evidenciado também pela diminuição dos conteúdos dos ácidos durante a produção dos vinhos.
A diminuição dos teores de fósforo com a vinificação dos mostos de abacaxi reforça a hipótese da precipitação do fosfato férrico. No entanto, os elevados teores de ferro encontrados nos vinhos de abacaxi podem ser devidos à doação deste elemento pelo uso da bentonite como clarificante. O cobre também apresentou teores mais baixos após a vinificação dos mostos de abacaxi, porém o teor deste elemento no vinho de abacaxi da cultivar Smooth cayenne (Tabela 2) estava acima de 0,4 mg. $\mathrm{L}^{-1}$, considerado limitante para a ocorrência da casse cúprica em vinhos de uvas (AMERINE; OUGH, 1974). É possível que, nos vinhos de abacaxi, os efeitos da presença do cobre sejam minimizados pela formação de complexos com o ácido cítrico, visto que este ácido é utilizado em vinhos de uvas para evitar as casses cúpricas e férricas (AMERINE; OUGH; 1974; AMERINE; BERG; CRUESS, 1972). Os teores de minerais encontrados nos vinhos de abacaxi estão de acordo com os relatados na literatura para vinhos de uvas (LAZOS; ALEXAKIS, 1989; RIZZON; SALVADOR, 1987; SOULIS; ARVANITOYANNIS; KAVLENTIS, 1989).

A Tabela 3 mostra a escala de pontos atribuídos às amostras de vinhos de abacaxi e a sua pontuação, cujas médias foram 6,60 e 6,96 (máximo de 9), respectivamente, para as cultivares Pérola e Smooth cayenne, correspondendo a Índices de Aceitabilidade (IA) de 73,3 e 77,3\%, indicando a aceitação desses vinhos, já que atingiram I.A. maiores que 70\% (TEIXEIRA; MEINERT; BARBETTA, 1987). Essa aceitação pode ser creditada à constituição química dessas bebidas, principalmente em função dos ácidos orgânicos formados durante a fermentação que, juntamente com o etanol, ajudaram na composição do sabor desses vinhos. Também é relevante a ausência de ácido acético nas referidas bebidas na sua aceitabilidade, já que a presença desse ácido em vinhos de uva prejudica sensorialmente.

A análise da variância da aceitabilidade dos vinhos de abacaxi (Quadro 1) indicou que não houve diferença significativa entre a aceitabilidade das duas cultivares $(\mathrm{p}=0,05)$.

Quadro 1. Análise da variância para a aceitabilidade dos vinhos de abacaxi das cultivares Pérola e Smooth cayenne.

\begin{tabular}{|lrrrcc|}
\hline F.V. & G.L. & S.Q. & Q.M. & Fc & $\mathrm{F}^{\circ}(0,05)$ \\
Tratamento & 1 & 4,63 & 4,63 & 0,05 & 3,84 \\
Resíduo & 144 & 12660,36 & 87,92 & & \\
\hline
\end{tabular}

Tabela 3. Frequências de Respostas (FR) para aceitabilidade de vinhos de abacaxi das cultivares Pérola e Smooth cayenne.

\begin{tabular}{|c|c|c|c|c|c|}
\hline \multirow[b]{2}{*}{ Escala } & \multirow[b]{2}{*}{ Pontuação (X) } & \multicolumn{2}{|c|}{ Pérola } & \multicolumn{2}{|c|}{ Smooth cayenne } \\
\hline & & $\mathrm{FR}^{1}$ & $F R \times X$ & $\mathrm{FR}^{1}$ & $\mathrm{FR} \times \mathrm{X}$ \\
\hline Gostei muitíssimo & 9 & 2 & 18 & 3 & 27 \\
\hline Gostei muito & 8 & 19 & 152 & 31 & 248 \\
\hline Gostei regularmente & 7 & 21 & 147 & 20 & 140 \\
\hline Gostei ligeiramente & 6 & 19 & 114 & 8 & 48 \\
\hline Indiferente & 5 & 5 & 25 & 4 & 20 \\
\hline Desgostei ligeiramente & 4 & 5 & 20 & 4 & 16 \\
\hline Desgostei regularmente & 3 & 2 & 6 & 3 & 9 \\
\hline Desgostei muito & 2 & 0 & 0 & 0 & 0 \\
\hline Desgostei muitíssimo & 1 & 0 & 0 & 0 & 0 \\
\hline Total & & 73 & 482 & 73 & 508 \\
\hline
\end{tabular}




\section{Conclusões}

A composição química dos vinhos de abacaxi mostrou-se semelhante à de vinhos de uvas quanto ao $\mathrm{pH}$, acidez e etanol, entre outros.

A formação de compostos voláteis pela levedura foi dependente da composição do mosto. Teores mais elevados de compostos fenólicos no vinho de abacaxi da cultivar Pérola foram relacionados com a maior intensidade de cor observada. Foi verificada diminuição nos teores de minerais e ácidos orgânicos durante a vinificação dos mostos de abacaxi.

Os vinhos de abacaxi das duas cultivares estudadas foram aceitos pelo painel sensorial utilizado.

\section{Referências bibliográficas}

ALIAN, A.; MUSSENGE, H. M. Utilization of pineapple waste for wine making. Journal of Science and Technology (Zambia), v. 1, p. 29-33, 1976.

AMERINE, M. A.; OUGH, C. S. Análisis de vinos y mostos. Zaragoza: Ed. Acribia, 1974. p. 121.

AMERINE, M. A.; BERG, H. W.; CRUESS, W. V. The Technology of wine making. Westport: [s.n], 1972. 801 p.

ARNOLD, R. M.; NOBLE, A. C.; SINGLETON, V. L. Bitterness and astringency of phenolics fraction of wine. Journal of Agricultural and Food Chemistry, v. 28, n. 3, p. 675-678, 1980.

ASSOCIATION OF OFFICIAL ANALYTICAL CHEMISTS - AOAC. Official methods of analysis. $15 \mathrm{ed}$. Virginia, 1986. $1141 \mathrm{p}$.

BENK, E. Uber tropische und subtropische weinahnliche Getranke. Alkohol-industrie, v. 6, p. 128-129, 1987.

BERTRAND, A. Formation des substances volatiles au cours de la fermentation alocoólique. In: COLLOQUE SOCIÉTÉ FRANÇAISE MICROBIOLOGY, Reims. p. 251-267, 1981.

BITTER, T.; MUIR, H. M. A modified acid uronic carbazole reaction. Analytical Biochemistry, v. 4, n. 4, p. 330-334, 1962.

BRASIL. Ministério da Agricultura e Reforma Agrária. Portaria n. 70, de 27 de julho de 1991. Diário Oficial da União, Brasília, 30 de agosto de 1991.

CANTARELLI, P. R. Tecnologia da transformação. In: Abacaxi Produção, pré-processamento e transformação agroindustrial. São Paulo: Governo do Estado de São Paulo, [19--]. p. 31-48.

COPPOLA, V. Precipitazione del ferro e del rame nei vini: cause e prevenzioni. Vignevini, v. 9, p. 19-21, 1982.

CHAVES, J. P. B. Avaliação sensorial de alimentos. Viçosa: Editora da Universidade Federal de Viçosa, 1980. 69 p.

DAUDT, C. E.; DURANTE, E. Adição de bentonite durante a vinificação de uvas brancas. 1 - Efeito sobre leveduras, clarificação, fermentação e sedimentos formados. Ciência e Tecnologia de Alimentos, v. 6, n. 1, p. 42-56, 1986.

DRYSDALE, G. S.; FLEET, G. H. Acetic acid bacteria in some Australian wines. Food Technology Australia, v. 37, n. 1, p. 17-20, 1985.

GNEKOW, B.; OUGH, C. S. Methanol in wines and most: sources and amounts. American Journal of Enology and Viticulture, v. 27, n. 1, p. 1-6, 1976.

GONZALEZ, M. J.; PARA, M. C.; AQUILAR, M. V. Elementos minerales en vinos. Estudio de interferencias organicas e inorganicas. Anales de Bromatologia, v. 40, p. 299-309, 1988.
GUINARD, J. X.; PANGBORN, R. M.; LEWIS, M. J. Preliminary studies on acidity-astringency interactions in model solutions and wines. Journal of the Science of Food and Agriculture, v. 37, n. 8, p. 811-817, 1986.

INTERNATIONAL UNION OF PURE AND APPLIED CHEMISTRY. Standard method for the analysis of oils, fats and derivatives. 6 ed. Oxiford: Pergamon Press, 1979.

KRAMLING, T. E.; SINGLETON, V.L. An estimate of the nonflavonoid phenols in the wine. American Journal of Enology and Viticulture, v. 20, n. 2, p. 86-92, 1969.

LAGE, M. A. et al. Metodo gas-chromatografico rapido para la evaluation directa de etanol y glicerina en vinos. Anales de Bromatologia, v. 41, n. 2, p. 373-381, 1989.

LAZOS, E. S.; ALEXAKIS, A. Metal ion content of some Greek wines. International Journal of Food Science \& Technology, v. 24, n. 1, p. 39-46, 1989.

LEE, C. Y.; COOLEY, H. J. Higher- alcohol contents in New York wines. American Journal of Enology and Viticulture, v. 32, n. 3, p. 244-246, 1981.

MALDONADO, O.; ROLZ, C.; CABRERA, S. Wine e vinegar production from tropical fruits. Journal Food Science, v. 40, n. 2, p. 262-265, 1972.

McCREADY, R. M.; McCOMB, E. A. Extraction and determination of total pectic material in fruits. Analytical Chemistry, v. 24, n. 12, p. 1986-1988, 1952.

MORALEDA, J. M. B. Aplicaciones en enologia del análisis por cromatografia líquida y gaseosa de ácidos, azúcares e poliálcoholes. Equipamentos e Tecnologia de Alimentos, v. 7, p. 83-88, 1988.

NOBLE, A. C. Bitterness and astringency in wine. In: Bitterness in food and beverages. Development in food science. 25 ed. New York: Elsevier, 1990. p. 145-158.

NOBLE, A. C.; BURSICK, G. F. The contribution of glycerol to perceved viscosity and sweetness in white wine. American Journal of Enology and Viticulture, v. 35, n. 2, p. 110-112, 1984.

NYKANEN, L. Formation and occurrence of flavour compounds in wine and distilled alcoholic beverages. American Journal of Enology and Viticulture, v. 37, n. 1, p. 84-96, 1986.

OHKUBO, T.; NOBLE, A. C.; OUGH, C. S. Evaluation of California Chardonnay wines by sensory and chemical analysis. Science des Aliments, v. 7, p. 200-214, 1984.

ONKARAYYA, H.; SINGH, H. Screening of mango varieties for Dessert and Madeira-style wines. American Journal of Enology and Viticulture, v. 35, n. 2, p. 63-67, 1984.

OUGH, C. S.; LEE, T. H. Effect of vineyard nitrogen fertilization level in the formation of some esters. American Journal of Enology and Viticulture, v. 32, n. 2, p. 125-127, 1981.

RIZZON, L. A. Composição química dos vinhos da microrregião homogênea vinicultura de Caxias do Sul (M.R.H. 311) Compostos voláteis. Bento Gonçalves: Embrapa Uva e Vinho, 1987. p. 1-4. (Comunicado Técnico, v. 5).

RIZZON, L. A.; SALVADOR, M. B. G. Teores de cátions dos vinhos da microrregião homogênea viticultora de Caxias do Sul (M. R. H. 311). Bento Gonçalves: Embrapa Uva e Vinho, 1987, p. 1-4. (Comunicado Técnico, v. 4).

ROBBS, P. G.; SANTANA, D. M. N.; GULARTE, J. L. Produção de etanol a partir de farinha de grãos de sorgo sacarino. Rio de Janeiro: Empresa Brasileira de Pesquisa Agropecuária, Centro de Tecnologia Agrícola e Alimentar, 1983. 32 p. (Documentos CTAA 003/83). 
SAN MIGUEL, T. L. Contenido de metanol y alcoholes superiores en vinos de Rioja. Anales del Instituto Nacional de Investigaciones Agrarias, v. 18, p. 145-152, 1982.

SHINOHARA, T.; WATANABE, M. Gas chromatographic analysis of higher alcohols and ethyl acetate in table wines. Agricultural and Biological Chemistry, v. 40, n. 12, p. 2475-2477, 1976.

SIMPSON, R. F. Some important aroma components of white wine. Food Technology in Australia, v. 30, p. 512-522, 1979.

SOMERS, T. C.; ZIEMELINS, G. Spectral evaluation of total phenolics componentes in Vitis vinifera: grapes and wines. Journal of The Science of Food and Agriculture, v. 36, n. 12, p. 1275-1284, 1985.
SOULIS, T.; ARVANITOYANNIS, I.; KAVLENTIS, E. Iron, coopper, manganese and zinc contents of some bottled and non-bottled greek wines. Science des Aliments, v. 9, p. 799-803, 1989.

TEIXEIRA, E.; MEINERT, E. M.; BARBETTA, P. A. Análise sensorial de alimentos. Florianópolis: Editora da Universidade Federal de Santa Catarina, 1987. p. 180.

WITH, L. M.; LODGE, N. Kiwifruit wine: production and evaluation. American Journal of Enology and Viticulture, v. 33, n. 4, p. 191-193, 1982.

ZOECKLEIN, B. W. et al. Production Wine Analysis. New York: A.V.I., 1989. p. 475. 